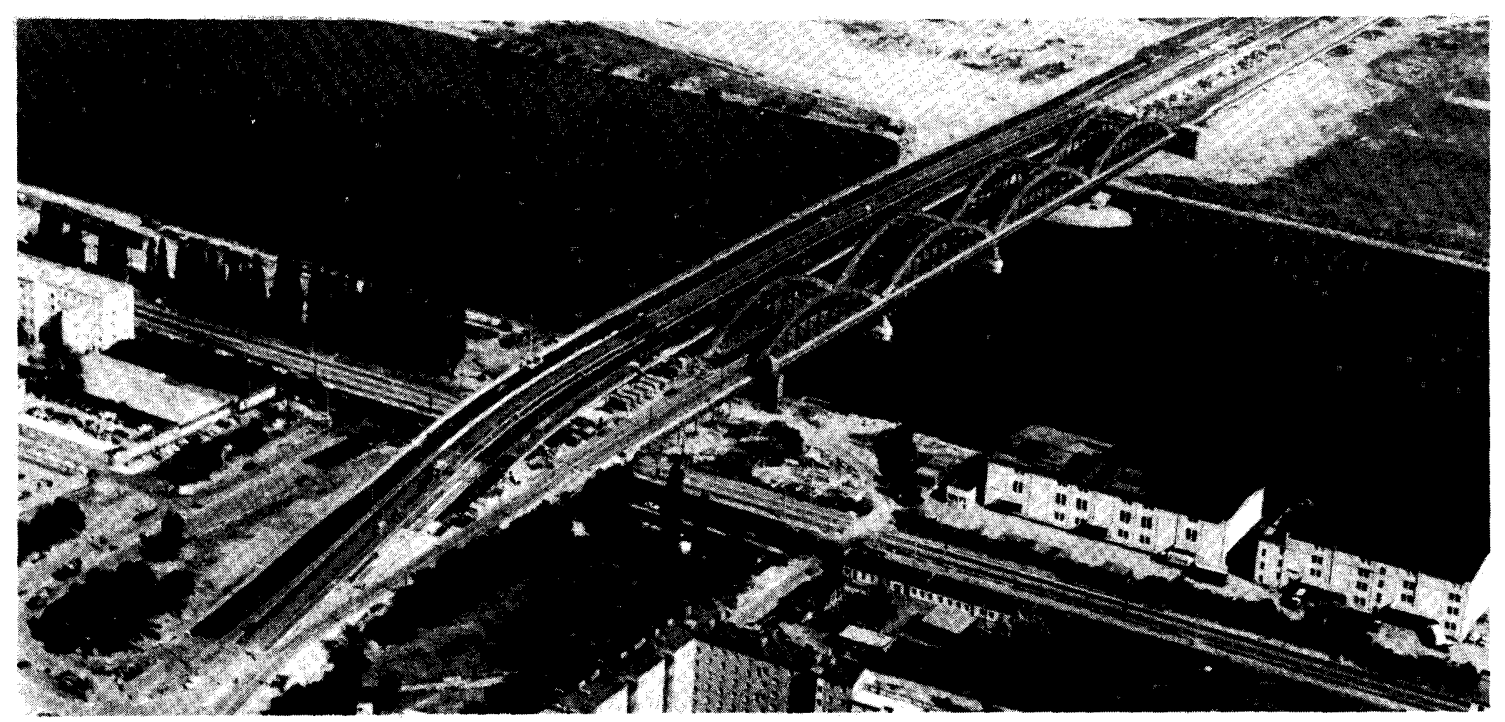

\title{
Nuevo puente de Floridsdorf en Viena - Austria
}

\section{sinopsis}

Esta obra se caracteriza por la gran rapidez de construcción, 18 meses, fecha récord mundial para este tipo de trabajos. Ello ha sido posible por la compenetración y perfecta organización de las empresas que más directamente han intervenido: Voest Alpine y Wagner.

Se describen el sistema de construcción, a base de grandes elementos metálicos y de hormigón; la fisonomia del puente, con sus partes a ambos lados del cauce y la que salva el curso propiamente dicho del rio; luces y alturas libres de los diferentes tramos; elevación y montaje, así como colocación de las piezas prefabricadas; cimentación, pilotaje y obras auxiliares.

Asimismo se enumeran las obras que quedan por hacer, aunque muchas de ellas están en vías de próxima terminación o muy avanzadas, bajo la dirección de la empresa Wiener Brückenbau $A G$.

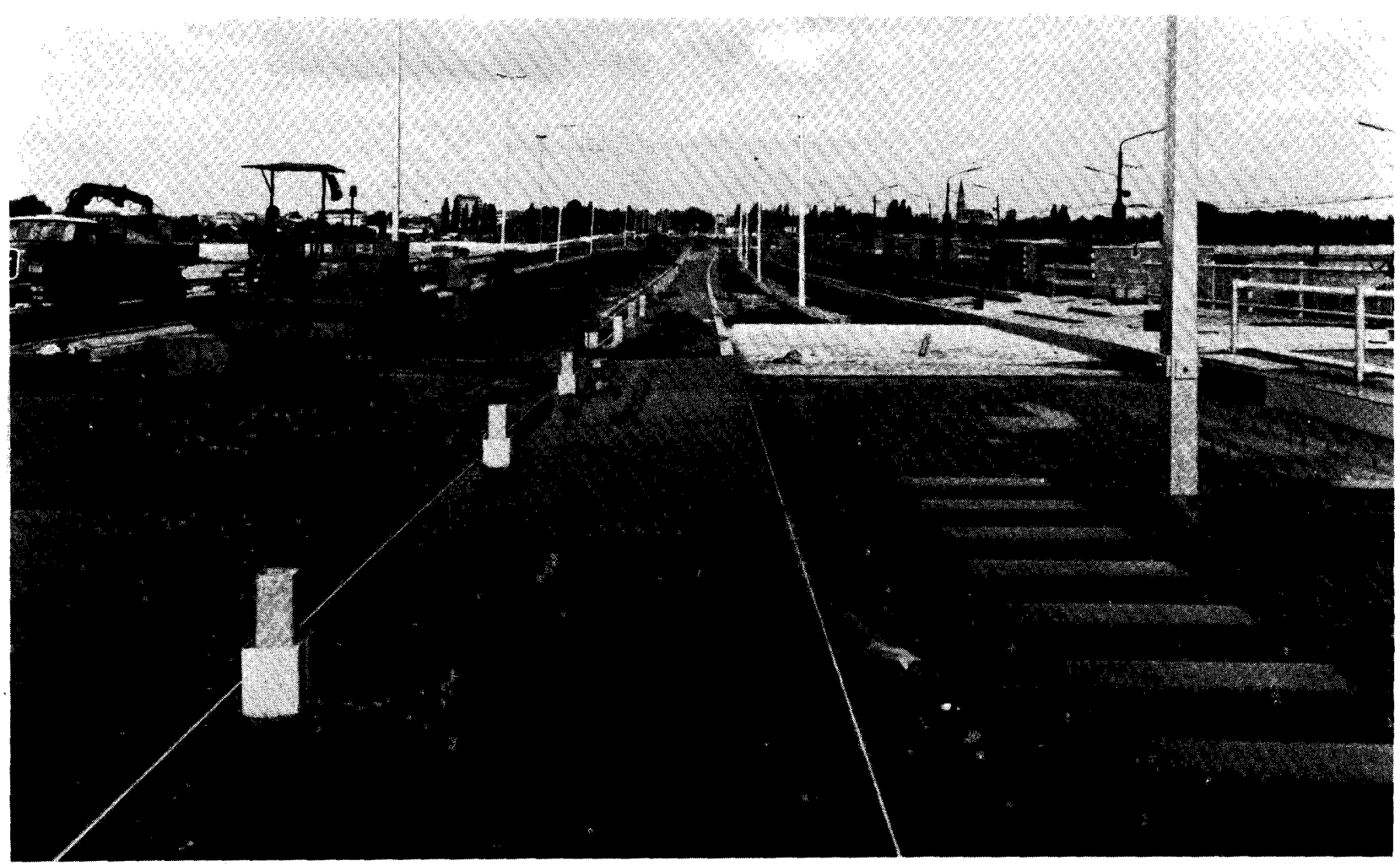




\section{sección transversal}

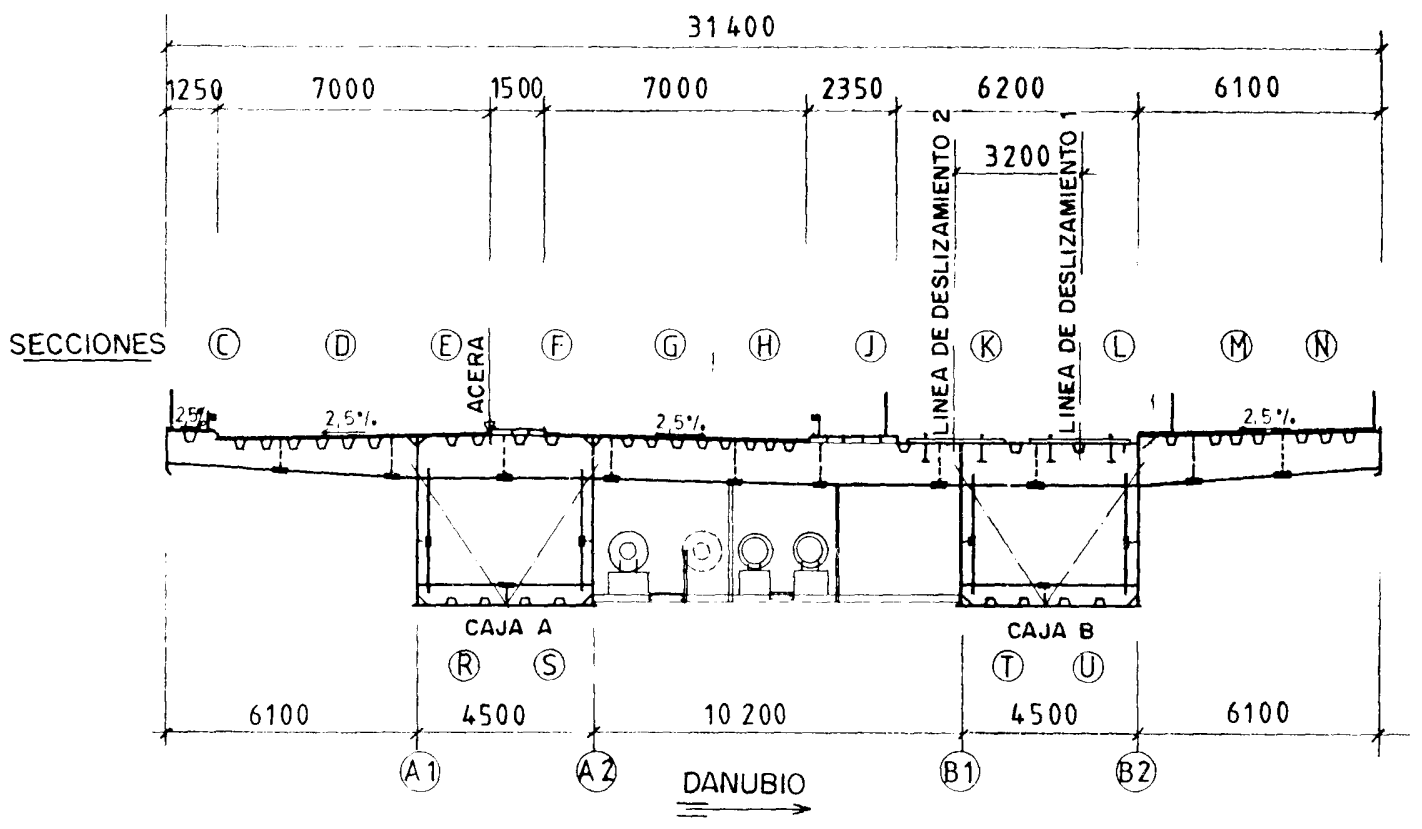

El tiempo empleado por el grupo de trabajo de construcciones de acero de los Puentes sobre el Danubio, en Viena, de sólo 18 meses, constituye un récord alcanzado por primera vez en todo el mundo. Hasta la fecha tales proyectos no se pudieron resolver en menos de 3 años. Por lo tanto se puede hablar de un récord mundial batido por las firmas Voest Alpine/Construcciones de Puentes de Viena y las Oficinas-Wagner. El reducido tiempo de construcción estaba fundamentalmente motivado por la posibilidad de una ejecución paralela de los trabajos.

Después de una planificación previa de 4 meses de duración (cálculo de la construcción con ayuda de grandes calculadoras electrónicas, acarreo de materiales, laminado de las chapas) se iniciaron los trabajos de taller en agosto de 1977. Mientras se estaban aún construyendo las pilas en el rio y los estribos del puente, se estaban realizando también, a plena marcha, los trabajos de la estructura horizontal de acero.

Otra ventaja consistia en el hecho de que el puente no se construia, como de costumbre, a base de pequeños elementos, sino que se unian grandes piezas de puente prefabricadas. Nueve meses después del comienzo de los trabajos, en enero de 1978, se inició el transporte de los elementos de acero ya montados en los talleres a la obra.

A mediados de abril de 1978 ya se habian realizado $217 \mathrm{~m}$ del puente sobre el río; a finales de mayo se produjo el montaje por flotación de los dos cajones de $116 \mathrm{~m}$ de longitud, con un peso de 570 t. El puente quedaba cerrado.

A partir de este momento ni siquiera una crecida de las aguas podía impedir la terminación del puente en el plazo previsto. Los habitantes de Viena pudieron utilizar nuevamente el puente de Floridsdorf a partir del mes de octubre de 1978.

Hasta la apertura al tráfico no habia transcurrido más que un periodo de 544 dias. El tiempo de trabajo empleado en la obra era de 950.000 horas. El número de horas normales ascendió a 630.000; las otras 320.000 horas correspondian a horas de trabajo en domingos y días festivos, asi como a horas nocturnas. Para la construcción del puente se emplearon $7.070 \mathrm{t}$ de acero y $32.000 \mathrm{~m}^{3}$ de hormigón. 


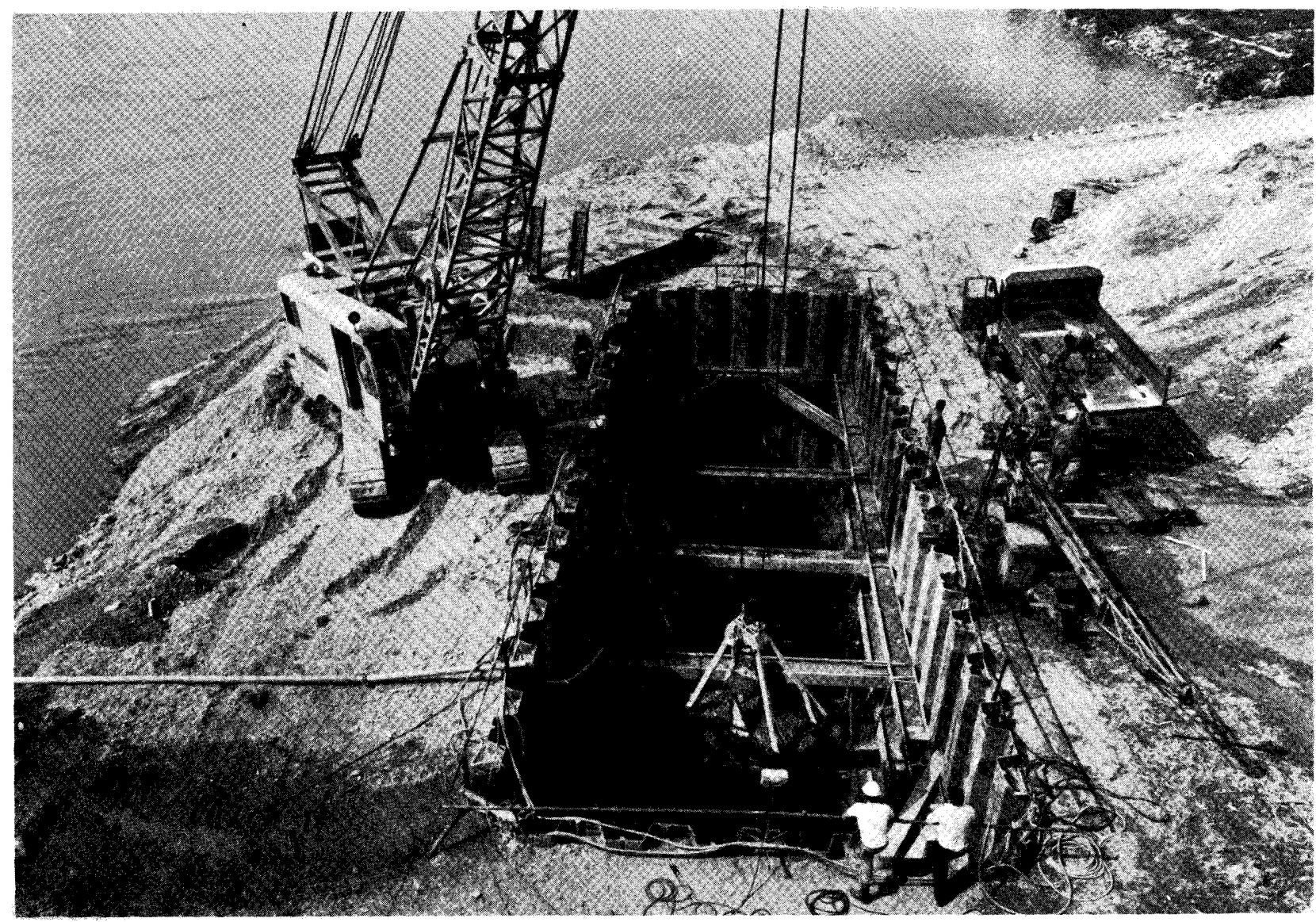

El citado puente dispone de cuatro carriles para el tráfico de vehiculos y una via para el tranvia, ya que se renunció a los carriles de autobús. Los expertos de tráfico pensaban que estos carriles serian innecesarios para las líneas interurbanas, ya que éstas no suelen pasar con frecuencia por el puente de Floridsdorf, y además son poco rentables, constituyendo incluso un peligro para la seguridad del tráfico debido a la situación lateral de las vías. El nuevo puente tiene doble capacidad que el puente antiguo, por lo que se puede esperar también una mejora considerable del tráfico en las cabezas del puente y la red viaria colindante, frente a la situación anterior.

En relación con la construcción técnica del puente de Floridsdorf puede decirse, que la obra se divide en dos partes:

- El propio puente sobre el río, con una longitud total de $332,5 \mathrm{~m}, \mathrm{y}$

- la estructura portante sobre el "Nuevo Danubio», que tiene una longitud de $215 \mathrm{~m}$.

El puente se presenta como una obra esbelta y elegante. Su altura es con las dos pilas, de 6,5 m; en el centro de la máxima luz es, sin embargo, de sólo 4,3 $\mathrm{m}$. La envergadura del puente entre las dos pilas es de $167,5 \mathrm{~m}$ y se alcanza un total, entre los estribos derecho e izquierdo, de $332,5 \mathrm{~m}$. La altura libre de navegación es, en una anchura de 130,4 m, de $8 \mathrm{~m}$.

Merece destacarse, especialmente, que el nuevo puente de acero sobre el Danubio tiene una longitud de $215 \mathrm{~m}$. La distancia entre apoyos es de $65,5+84+65,5 \mathrm{~m}$. En dirección aguas arriba se encuentra un paso de peatones de 1,24 $\mathrm{m}$ de anchura $\mathrm{y}$, aguas abajo, otro de $6,10 \mathrm{~m}$. La construcción se parece a la de cualquier otro puente sobre un río, así como el tablero sobre el "Nuevo Danubio", siendo sin embargo la anchura del puente, por razones técnicas de circulación, de 37,40 metros. 


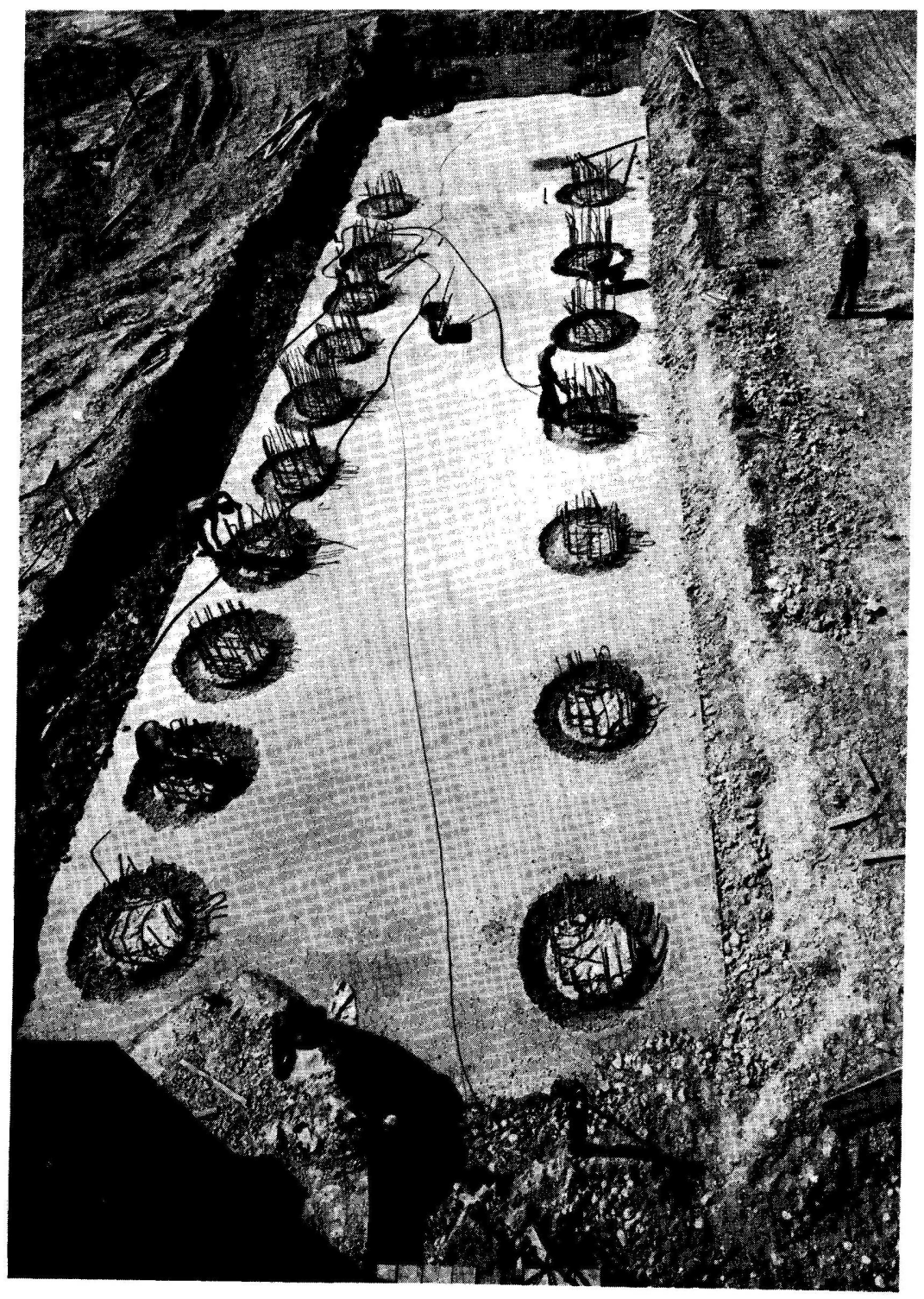

La sección transversal del nuevo puente consta de dos tableros de $7 \mathrm{~m}$ de anchura y una mediana de $1,50 \mathrm{~m}$. Para el tranvia se ha previsto un espacio de $6,20 \mathrm{~m}$. Entre los carriles y el tranvia hay una isla protectora, contra el tráfico, de $2,35 \mathrm{~m}$ de anchura. En el puente sobre el Nuevo Danubio se aumentó el tablero aguas abajo a una anchura de 10 hasta $15 \mathrm{~m}$; la isla del tranvía varía en su

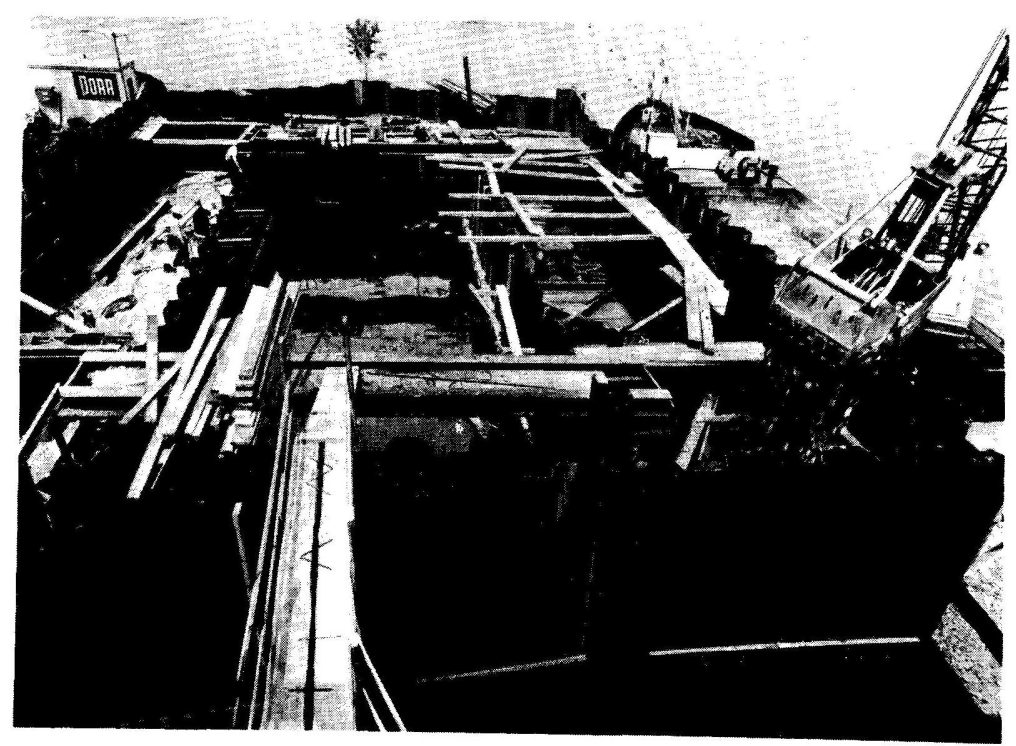



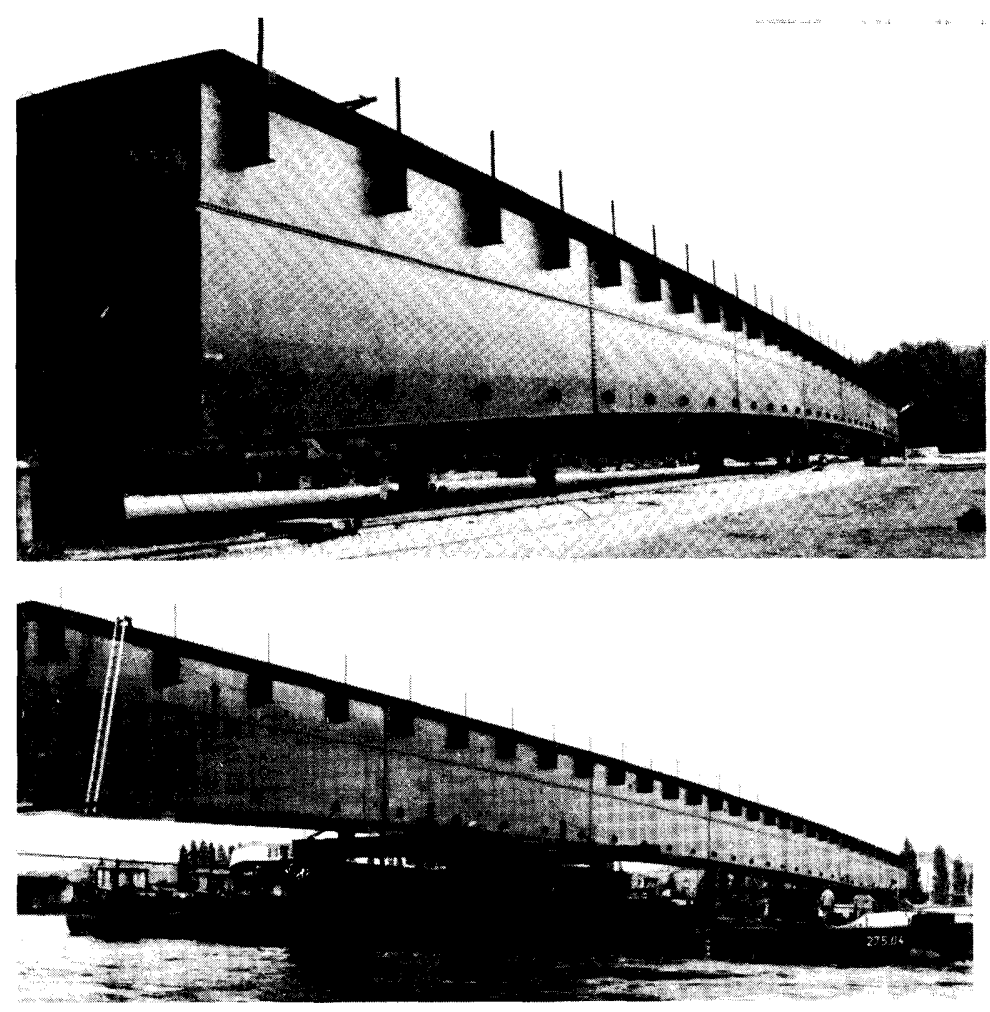

La parte principal desde el estribo a este pilote de montaje se montó por detrás del estribo; se desplazó después por medio de una auto-grúa y se colocó en su posición definitiva.

Este procedimiento correspondia respectivamente a un cajón uniéndose los dos, con una placa, por medio de un carro de construcción en saliente. Posteriormente se unian las siguientes partes de cajón (desde el pilote de montaje hasta las pilas) en el lugar de avance, a fin de colocarlas con ayuda de una grúa flotante. Esta operación tuvo que realizarse también individualmente para cada cajón.

\section{montaje}
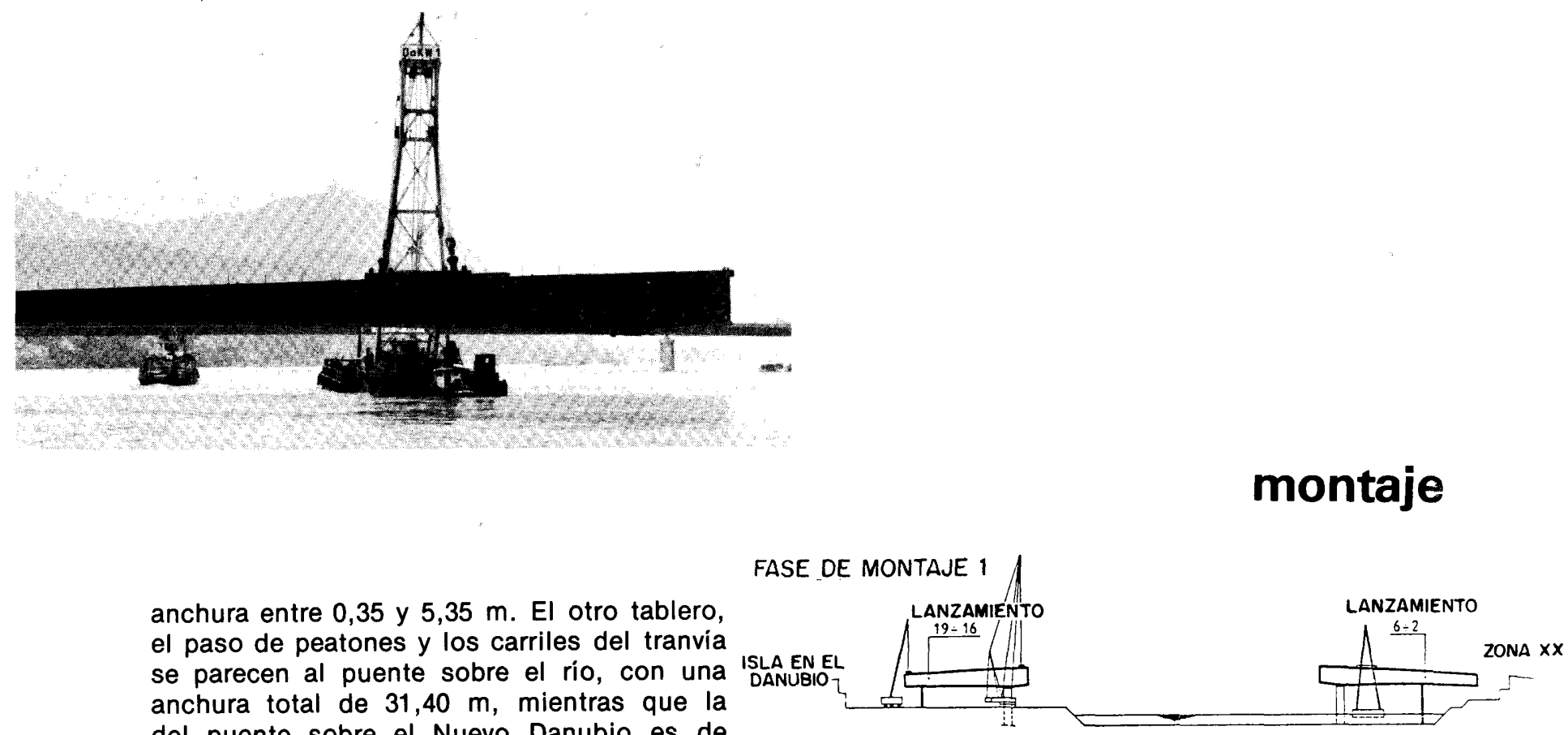

anchura entre 0,35 y $5,35 \mathrm{~m}$. El otro tablero, el paso de peatones y los carriles del tranvia se parecen al puente sobre el rio, con una anchura total de $31,40 \mathrm{~m}$, mientras que la del puente sobre el Nuevo Danubio es de $37,40 \mathrm{~m}$, con un ensanchamiento de aproximadamente $1 \mathrm{~m}$ en el estribo de Floridsdorf.

Los carriles del tranvia se alojan en unas traviesas situadas sobre una placa ortótropa. No existe ningún lecho de balasto. La altura desde el borde superior de los rieles al borde superior de la chapa de la calzada es de $32 \mathrm{~cm}$. La sección transversal se compone en ambos puentes de cajas huecas bicelulares unidas a la placa ortótropa. Las cajas tienen una anchura de $4,50 \mathrm{~m}$, la distancia entre las almas interiores de las cajas es en el puente del río de $10,20 \mathrm{~m}$, en el otro de $16,20 \mathrm{~m}$. Para el montaje del puente del río se eligió una combinación de premontaje sobre tierra de unos elementos y montaje por flotación de otros. Respectivamente en el centro de los dos huecos laterales se dispuso un pilote de montaje.

FASE DE MONTAJE 2

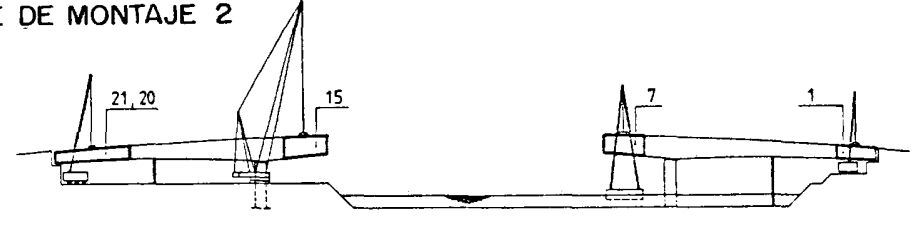

FASE DE MONTAJE 3

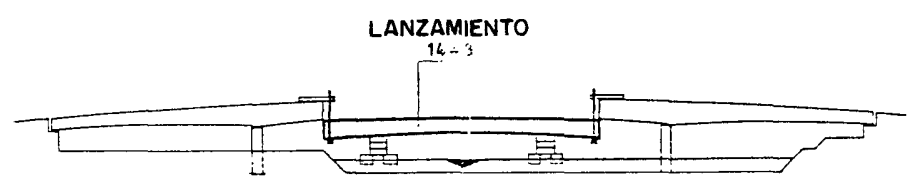

FASE DE MONTAJE 4

LANZA- $21,20,19,18,17,16,15,14,13,12,11,10,9,8,7,6,5, T^{6}, T^{3}, T^{2}, 1$

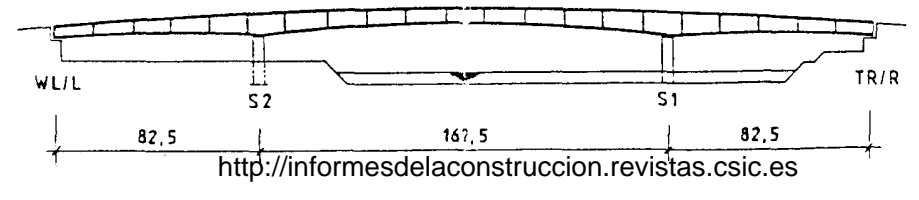



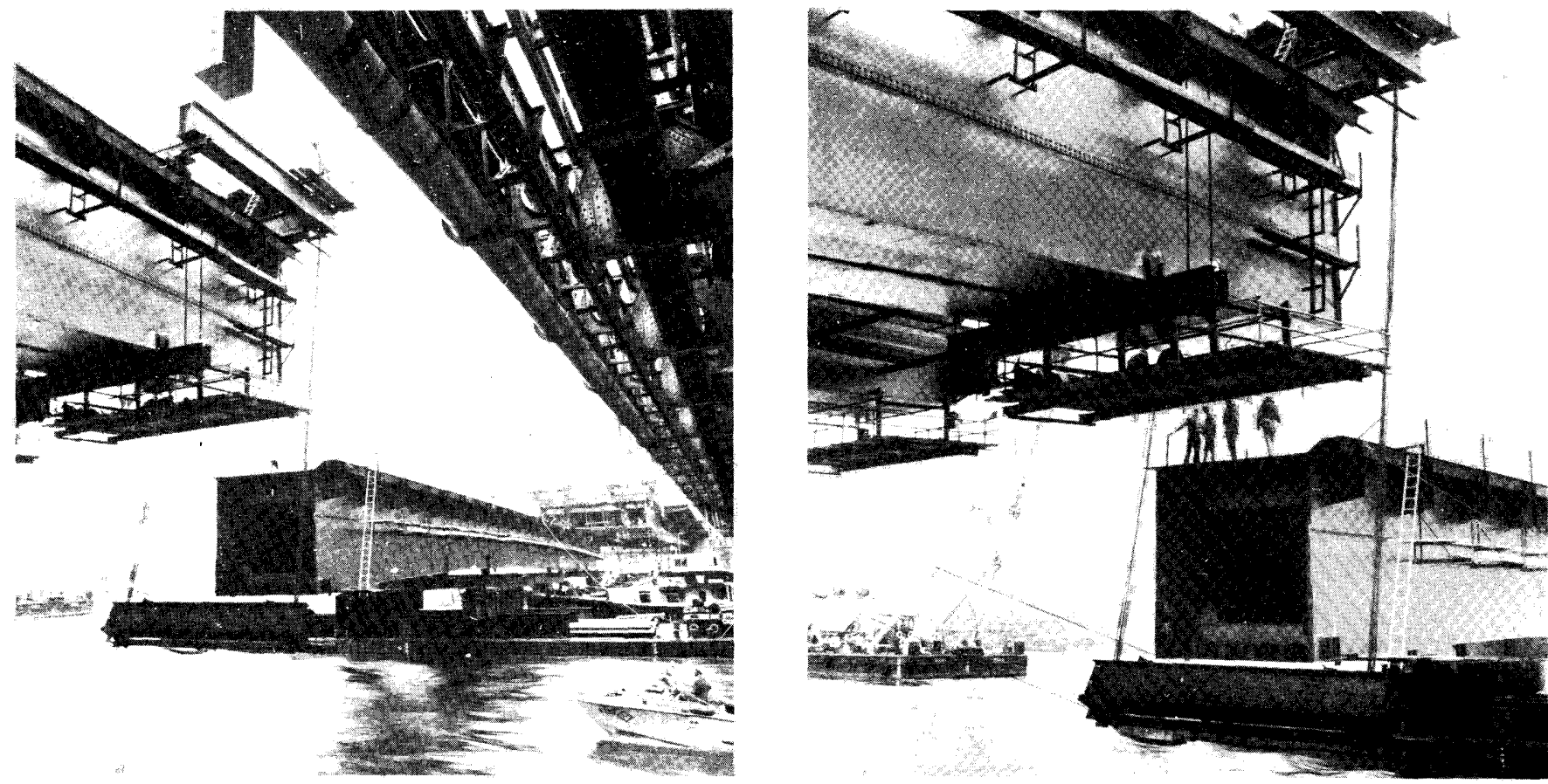

Las dos partes restantes eran los cajones de la parte central, las cuales constituian el trabajo más difícil del montaje. Con una longitud de $116,2 \mathrm{~m}$ pesaban, más o menos, $550 \mathrm{t}$ y se montaron, aguas arriba, en un lugar de montaje a $300 \mathrm{~m}$ de distancia. La grúa flotante no podia emplearse para nada, ni siquiera en caso de colocarlas separadas a lo largo, como las partes derechas. Por lo tanto hubo que tirar de los cajones transversalmente, con respecto al río, hasta que un extremo sobresalia sobre el agua. En este momento se colocaba por debajo una gabarra rellena de agua. Vaciando esta gabarra, por medio de una bomba, se elevaba y, con ella, el extremo del cajón hueco. Una vez trasladada la gabarra en dirección al centro del río se procedió de la misma manera con el segundo extremo de la estructura portante.

Después de montar por flotación estos dos gigantes, se produjo su elevación por medio de prensas hidráulicas. Posteriormente pudo tenderse el puente mediante el montaje de piezas de ajuste. Después del montaje de los brazos en voladizo, hacia los extremos del puente, quedaba terminada la construcción de acero del puente sobre el rio.
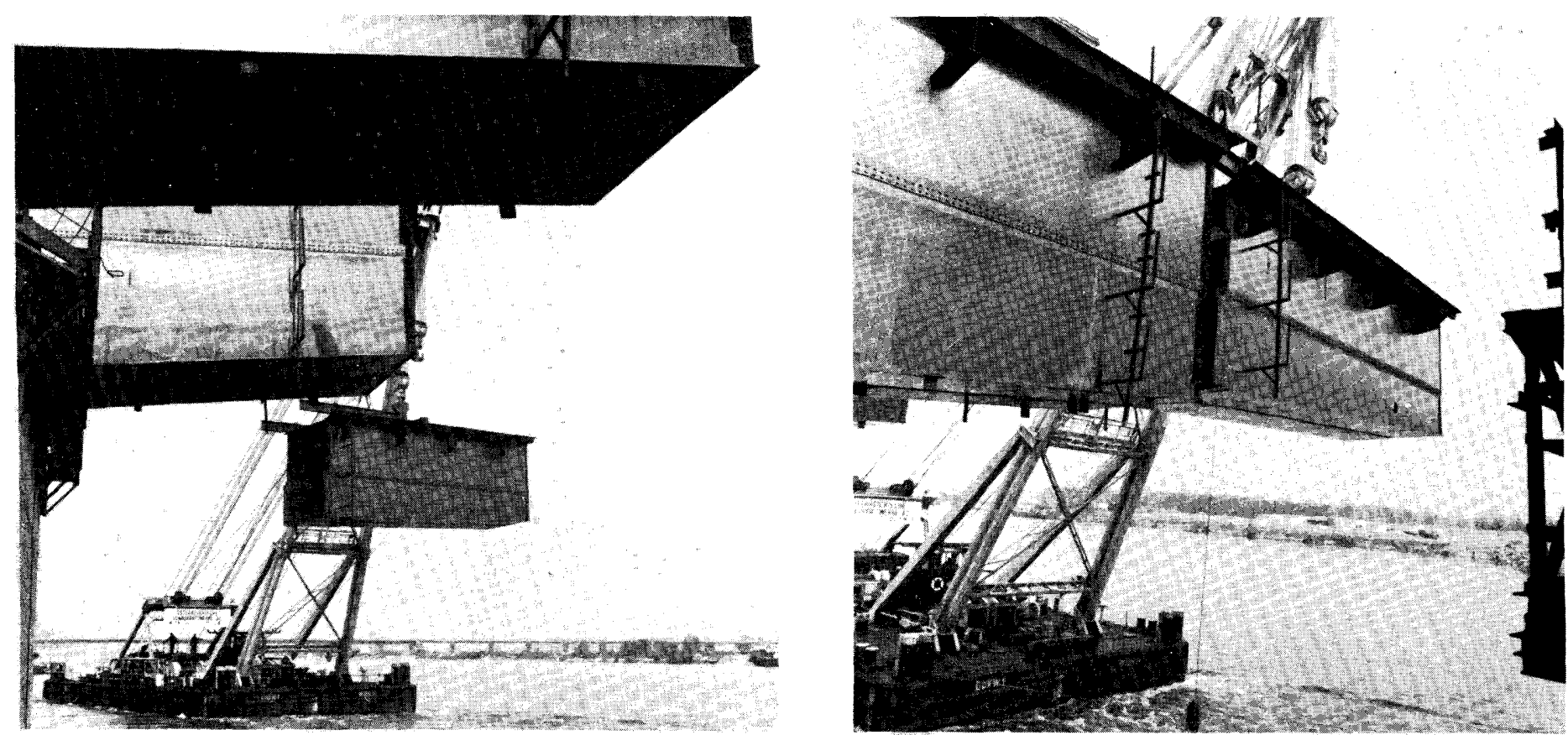
Las partes de cajón de los laterales se montaron, a su vez, en el suelo y se colocaron en su posición definitiva por medio de unas grúas móviles. También los cajones de la parte central se montaron en el suelo y se elevaron, como en el cajón anterior, por medio de unos dispositivos elevadores hidráulicos con aceros tensados; y se unieron con el puente ya montado.

El puente del río se apoya sobre doce puntos; el puente del lecho descarga sobre ocho apoyos. En ambos casos se prevé solamente un apoyo fijo, los demás son móviles. Esto permite un cierto movimiento a la estructura portante.

El desagüe del puente se lleva a cabo a través de dos conductos dispuestos a lo largo de la estructura portante del puente y las entradas se sitúan en la placa ortótropa.

Para el alojamiento de los tubos se han dispuesto, a lo largo del puente, unas suspensiones y ramales de maniobra situados entre los cajones de los soportes principales. Allí se encuentran los cables eléctricos de baja y alta tensión. En cada cajón existe, además, un paso de servicio.

La parte de la construcción del puente, que probablemente ofrecía mayores riesgos, era el montaje. Estos trabajos se realizaron aún con relativa facilidad en el caso del puente sobre el Nuevo Danubio. Las pariés de cajón prefabricado de los laterales, se montaron en el suelo y se colocaron en su posición definitiva con ayuda de unas grúas móviles. Los cajones se alojaron en los estribos y en los pilares. A continuación se montaron las chapas de las pistas, entre los cajones. Las almas principales y transversales se atornillaron por el procedimiento-HV. Todas las demás uniones fueron soldadas.

También se montaron en el suelo los cajones de la parte central elevándose por partes y uniéndose después al puente ya montado. Posteriormente se montaron las chapas de las pistas en la parte central del puente, así como todas las piezas en voladizo.

Mucho más complicado y costoso resultó el montaje del puente sobre el río. Sólo el equipo flotante representaba un valor de 10 millones de chelines. En este caso se decidió realizar la operación a base de combinar un montaje previo en tierra y otro montaje por flotación.

Todo el puente sobre el río consta de 21 elementos en cajón hueco. Cada uno tiene una longitud de 16 a $17 \mathrm{~m}$ y pesa, según el tamaño, de 20 a $30 \mathrm{t}$.

En el transcurso de la construcción nueva del puente de Floridsdorf fueron también necesarios importantes trabajos de excavación. Una condición previa importantísima, para el plazo total tan reducido, consiste en la construcción de las dos pilas.

También en este caso pudieron aprovecharse las experiencias obtenidas en la construcción de los puentes adicionales del Reichsbrücke. Alli, y por primera vez en Austria, no se apilaron pequeñas islas para la construcción de las pilas, sino que se construyeron unas islas de acero. IDel mismo modo se procedió en el puente de Floridsdorf.

La isla de acero se construyó en tierra y se desplazó después. Desde la misma se pudieron colocar, con bastante independencia del nivel del agua, los pilotes de perforación, en un número total de 40 a aproximadamente $15 \mathrm{~m}$ por debajo de la solera del río. Este grupo de pilotes se bajó y cubrió en el fondo del río con una placa de hormigón armado de 1,7 $\mathrm{m}$ de espesor, sobre la cual se dispuso otro grupo de pilotes. Los $6 \mathrm{~m}$ superiores de los pilotes, toda la placa de base y las capas exteriores de los pilotes se construyeron a base de hormigón resistente a la abrasión.

Cada uno de los dos pilotes del río tiene una longitud de $21 \mathrm{~m} \mathrm{y} 4$ de anchura. El derecho se encuentra a $60 \mathrm{~m}$ de distancia de la orilla, el izquierdo a $40 \mathrm{~m}$; el paso central tiene una anchura de $167 \mathrm{~m}$. Como ya se dijo antes, los asi llamados pilotes-guia se componen, cada uno, de un tubo de acero de 1,2 m de diámetro, cuyo espesor de pared es de $12 \mathrm{~mm}$. Baja $17 \mathrm{~m}$ por debajo de la solera del río y está relleno de hormigón armado.

Los trabajos a realizar en las dos pilas del rio se efectuaron en turnos de dia y noche, durante un periodo ininterrumpido de 5 meses.

De los demás trabajos a realizar en esta gran obra quedaban por terminar las construcciones subterráneas, dirigidas por la firma Porr-Baugesellschaft $A G$, Viena, las cuales se indican a continuación y que, en gran parte, están concluidas: 
- un túnel para peatones en la plaza Engelsplatz;

- la rampa de Engelsplatz de acceso al puente de hormigón pretensado sobre el muelle y las vias del tren;

- trabajos de relleno y apilamiento para los diques de protección, contra las crecidas de las aguas y para el dique de la isla en el Danubio;

- pilas y estribos para el puente sobre el Nuevo Danubio;

- puente de hormigón pretensado sobre las vías del tren, a orillas del Danubio;

- la rampa de la calle principal de Floridsdorf;

- trabajos complementarios en todos los puentes y rampas.

Como ya se dijo antes, todos estos trabajos se encuentran en su fase final. Por añadidura se puede decir que la firma vienesa Wiener Brückenbau AG dirigía los trabajos de construcción metálica.

\section{W. Schmidt}

\section{résumé}

NOUVEAU PONT DE FLORIDSDORF A VIENNE - AUTRICHE

:Cet ouvrage est caractérisè pour la grande rapidité de construction, 18 mois, temps record mondial pour cette sorte de travaux. Cela a été possible pour la compenétration et l'organisation parfaite des entreprises qui $y$ ont participé le plus directement; Voest Alpine et Wagner.

On décrit le système de construction, constitué par des grands éléments métalliques et en béton, la physionomie du pont, avec ses parties sur les rives du cours d'eau et celle qui franchit le cours d'eau proprement dit, les portées et les hauteurs libres de différents tronçons, la mise en place des pièces préfabriquées, la fondation, le pilotis et les travaux auxiliaires.

On énumère également les travaux qui sont encore à exécuter, bien que bon nombre d'entre eux sont très avancés ou seront bientôt terminés, sous la direction de l'entreprise Wiener Brückenbau AG.

\section{summary}

NEW FLORIDSDORF BRIDGE IN VIENNA - AUSTRIA

This work was characterised by the great speed with which it was built, 18 months, a world record for this type of job. This was possible thanks to the compenetration and perfect organisation of the companies who intervened most directly: Voest Alpine and Wagner.

The construction system is described, made of large metal and concrete elements; the physiognomy of the bridge, with its parts on both sides of the riverbed and that which covers the real course of the river; spans and free heights of the different stretches; elevations and assembly, as well as laying prefabricated parts; foundations, piling and ancillary work.

Work still to be done is also enumerated although many of the jobs will shortly be completed or are in a very advanced stage, completed or are in a very advanced stage, Wrückenbau AG.

\section{zusammenfassung NEUE FLORIDSDORFER-BRÜCKE IN WIEN - ÖSTERREICH}

Dieses Bauwerk zeichnet sich durch dessen rasche Ausführung aus. Es wurde in 18 Monaten erstellt, was einen Weltrekord für diese Art von Arbeiting darstellt. Dies war durch die gute Zusammenarbeit und einwandfreie Organisation der unmittelbar beteiligten Unternehmen, Voest Alpine und Wagner, möglich.

Es wird das Bausystem aufgrund grosser Metall- und Betonteile beschrieben; die Gestaltung der Brücke mit ihren Teilen beidseits des Flussbettes und dem den Strom überquerenden Teil; Spannweiten und freie Höhen der verschiedenen Abschnitte; Heben und Montage, sowie Anbringung der vorgefertigten Teile; Fundamentierung, Pfeiler und Hilfsbauten.

Desgleichen werdwn die noch unter der Leitung der Wiener Brückenbau AG auszuführenden Arbeiten erwähnt, wobei zwar viele derselben bald beendet sein werden oder doch schon sehr fortgeschritten sind. 